\title{
OSTROWSKI TYPE INEQUALITY USING FIVE STEP WEIGHTED KERNEL
}

\section{SOFIAN OBEIDAT ${ }^{1, *}$, MUHAMMAD AMER LATIF ${ }^{1}$ AND ATHER QAYYUM ${ }^{2}$}

\author{
${ }^{1}$ Department of Basic Sciences, \\ Deanship of Preparatory Year, University of Hai'l, Saudi Arabia \\ ${ }^{2}$ Department of Mathematics, Institute of Southern Punjab Multan, Pakistan
}

${ }^{*}$ Corresponding author: obeidatsofian@gmail.com

\begin{abstract}
The purpose of this paper is to establish weighted version of Ostrowski type integral inequalities. The inequalities are obtained by using a newly developed special type of five steps weighted kernel. The introduction of this new Kernel gives some new error bounds for various quadrature rules. Applications for Cumulative Distributive Functions are considered.
\end{abstract}

\section{Introduction}

The field of inequalities have applications in most of the domains of Mathematics. The importance of mathematical inequalities has increased during the past few decades and now it is studied as a separate branch of Mathematics. A number of research papers and books have been written on inequalities and their applications (see for instance [9]- [13]). In many practical problems, it is important to bound one quantity by another quantity. The classical inequalities such as Ostrowski's inequality is very useful for this purpose. Ostrowski type inequalities have immediate applications in numerical integration, optimization theory, statistics and integral operator theory.

In 1938, Ostrowski [8] discovered the following useful integral inequality.

Received 2019-02-14; accepted 2019-03-07; published 2019-05-01.

2010 Mathematics Subject Classification. Primary 26D15, 26D20; Secondary 26D99.

Key words and phrases. Ostrowski inequality; numerical Integration; composite quadrature rule; cumulative distributive function.

(C) 2019 Authors retain the copyrights of their papers, and all open access articles are distributed under the terms of the Creative Commons Attribution License. 
Theorem 1.1. Let $f:[a, b] \rightarrow R$ be continuous on $[a, b]$ and differentiable on $(a, b)$, whose derivative $f^{\prime}:(a, b) \rightarrow R$ is bounded on $(a, b)$, i.e.

$$
\left\|f^{\prime}\right\|_{\infty}=\sup _{t \in[a, b]}\left|f^{\prime}(t)\right|<\infty
$$

then for all $x \in[a, b]$

$$
\left|f(x)-\frac{1}{b-a} \int_{a}^{b} f(t) d t\right| \leq\left[\frac{1}{4}+\left(\frac{x-\frac{a+b}{2}}{b-a}\right)^{2}\right](b-a)\left\|f^{\prime}\right\|_{\infty} .
$$

We mention another inequality called Grüss inequality [6] which is stated as the integral inequality that establishes a connection between the integral of the product of two functions and the product of their individual integrals. Mathematically, it is described as follows:

$$
\begin{aligned}
& \left|\frac{1}{b-a} \int_{a}^{b} f(x) g(x) d x-\frac{1}{b-a} \int_{a}^{b} f(x) d x \cdot \frac{1}{b-a} \int_{a}^{b} g(x) d x\right| \\
\leq & \frac{1}{4}(\Phi-\varphi)(\Gamma-\gamma),
\end{aligned}
$$

where

$$
\varphi \leq f(x) \leq \Phi \text { and } \gamma \leq g(x) \leq \Gamma,
$$

for all $x \in[a, b]$. The constant $\frac{1}{4}$ is sharp in (1.2) .

In [4], Dragomir and Wang combined Ostrowski and Grüss inequality to give a new inequality which they named Ostrowski-Grüss type inequalities.

In [2], Barnett et.al proved some Ostrowski type inequality and generalized the trapezoidal inequality. Dragomir [3] and Liu [5] established some companions of Ostrowski type integral inequalities.

Recently, Qayyum et. al. [15] proved some Ostrowski type inequalities, they obtained their results by using kernel with five steps. In this paper, we will present the weighted version of the results obtained by Qayyum et. al. [15].

Throughout the present paper, a weight function (or density function) over some interval $[a, b]$, where $-\infty<a<b<\infty$, is a function $w:[a, b] \longrightarrow[0, \infty)$ with $0<\int_{a}^{b} w(t) d t<\infty$ 


\section{Main Results}

Definition 2.1. Let $-\infty<a<b<\infty$. Let $w$ be a weight function over $[a, b]$. The 5-step linear kernel with respect to $w$ is denoted by $P_{w}$, and is defined as follows:

$$
P_{w}(x, t)=\left\{\begin{array}{cc}
\int_{a}^{t} w(u) d u, & t \in\left[a, \frac{a+x}{2}\right] \\
\frac{3}{4} \int_{a}^{t} w(u) d u+\frac{1}{4} \int_{b}^{t} w(u) d u, & t \in\left(\frac{a+x}{2}, x\right] \\
\frac{1}{2} \int_{a}^{t} w(u) d u+\frac{1}{2} \int_{b}^{t} w(u) d u, & t \in(x, a+b-x] \\
\frac{1}{4} \int_{a}^{t} w(u) d u+\frac{3}{4} \int_{b}^{t} w(u) d u, & t \in\left(a+b-x, \frac{a+2 b-x}{2}\right] \\
\int_{b}^{t} w(u) d u, & t \in\left(\frac{a+2 b-x}{2}, b\right]
\end{array}\right.
$$

for $x \in\left[a, \frac{a+b}{2}\right]$ and $t \in[0,1]$.

The following lemma will be used repeatedly throughout the present paper.

Lemma 2.1. Let $-\infty<a<b<\infty$. For a weight function $w$ over $[a, b]$, the identity

$$
\begin{aligned}
& \int_{a}^{b} P_{w}(x, t) f^{\prime}(t) d t \\
= & \frac{1}{4}\left[\int_{a}^{b} w(t) d t\right]\left[f(x)+f(a+b-x)+f\left(\frac{a+x}{2}\right)+f\left(\frac{a+2 b-x}{2}\right)\right]-\int_{a}^{b} w(t) f(t) d t,
\end{aligned}
$$

holds for all $x \in\left[a, \frac{a+b}{2}\right]$ and $t \in[0,1]$.

Proof: Obvious.

Lemma 2.2. Let $-\infty<a<b<\infty$, and $w$ be a weight function over $[a, b]$. If $w$ is symmetric about $\frac{a+b}{2}$, then

$$
\int_{a}^{b} P_{w}(x, t) d t=0 .
$$

Proof: Since $w$ is symmetric about $\frac{a+b}{2}$,

$$
\begin{aligned}
\int_{a}^{b} t w(t) d t & =\int_{a}^{b}(a+b-t) w(a+b-t) d t \\
& =\int_{a}^{b}(a+b-t) w(t) d t
\end{aligned}
$$

which implies that

$$
\int_{a}^{b} t w(t) d t=\frac{b+a}{2} \int_{a}^{b} w(t) d t
$$


Using Identity (2.2) with $f(t)=t$, we get that

$$
\int_{a}^{b} P_{w}(x, t) d t=\frac{b+a}{2} \int_{a}^{b} w(t) d t-\int_{a}^{b} t w(t) d t=0 .
$$

The converse of Lemma 2.2 is not correct in general as shown in the following example.

Example 2.1. For $-\infty<a<b<\infty$, let $h(x)$ and $w(x)$ be defined as:

$$
\begin{aligned}
h(x) & =-2+\frac{10}{3}\left(\frac{x-a}{b-a}\right), \\
w(x) & =\frac{1}{9}(h(x))^{2}+\frac{1}{27}(h(x))^{3}, \text { for } x \in[a, b] .
\end{aligned}
$$

Clearly, $w$ is not symmetric about $\frac{a+b}{2}$. On the other hand, note that

$$
\int_{a}^{b} w(t) d t=\frac{58}{729}(b-a)
$$

and

$$
\int_{a}^{b} t w(t) d t=\frac{29}{729}\left(b^{2}-a^{2}\right)
$$

which implies that

$$
\int_{a}^{b} t w(t) d t=\frac{a+b}{2} \int_{a}^{b} w(t) d t
$$

Thus,

$$
\int_{a}^{b} P_{w}(x, t) d t=0
$$

Now with the help of Lemma 2.1, we state and prove some theorems in the following subsections.

\subsection{The $L^{1}$ Case.}

Theorem 2.1. Let $-\infty<a<b<\infty$ and $f:[a, b] \rightarrow \mathbb{R}$ be a differentiable function on $(a, b)$. Suppose that $w$ is a weight function over $[a, b]$ with $\int_{a}^{b} t w(t) d t=\frac{b+a}{2} \int_{a}^{b} w(t) d t$. If $f^{\prime} \in L^{1}[a, b]$ and $D_{1} \leq f^{\prime}(t) \leq D_{2}$, for all $t \in[a, b]$, where $D_{1}, D_{2}$ are constants, then the inequality

$$
\begin{aligned}
& \left|\frac{1}{4}\left(\int_{a}^{b} w(t) d t\right)\left(f(x)+f(a+b-x)+f\left(\frac{a+x}{2}\right)+f\left(\frac{a+2 b-x}{2}\right)\right)-\int_{a}^{b} w(t) f(t) d t\right| \\
\leq & \frac{1}{2}\left[\int_{a}^{b} w(t) d t\right]\left(D_{2}-D_{1}\right)(b-a)
\end{aligned}
$$

holds for all $x \in\left[a, \frac{a+b}{2}\right]$. 
Proof. Note that for $t \in[0,1]$ and $x \in\left[a, \frac{a+b}{2}\right]$, we have

$$
\int_{b}^{a} w(t) d t \leq P_{w}(x, t) \leq \int_{a}^{b} w(t) d t
$$

Using Identity (2.2) with $f(t)=t$ and the fact that $\int_{a}^{b} t w(t) d t=\frac{b+a}{2} \int_{a}^{b} w(t) d t$, we get that

$$
\int_{a}^{b} P_{w}(x, t) d t=0
$$

which implies that

$$
\frac{1}{b-a} \int_{a}^{b} P_{w}(x, t) f^{\prime}(t) d t=\frac{1}{b-a} \int_{a}^{b} P_{w}(x, t) f^{\prime}(t) d t-\frac{1}{(b-a)^{2}} \int_{a}^{b} P_{w}(x, t) d t \int_{a}^{b} f^{\prime}(t) d t
$$

Applying Grüss Inequality, we get that

$$
\begin{aligned}
& \left|\frac{1}{b-a} \int_{a}^{b} P_{w}(x, t) f^{\prime}(t) d t\right| \\
= & \left|\frac{1}{b-a} \int_{a}^{b} P_{w}(x, t) f^{\prime}(t) d t-\frac{1}{(b-a)^{2}} \int_{a}^{b} P_{w}(x, t) d t \int_{a}^{b} f^{\prime}(t) d t\right| \\
\leq & \frac{1}{4}\left(\int_{a}^{b} w(t) d t-\int_{b}^{a} w(t) d t\right)\left(D_{2}-D_{1}\right) . \\
= & \frac{1}{2}\left[\int_{a}^{b} w(t) d t\right]\left(D_{2}-D_{1}\right) .
\end{aligned}
$$

By Lemma 2.1,

$$
\begin{aligned}
& \int_{a}^{b} P_{w}(x, t) f^{\prime}(t) d t \\
= & \frac{1}{4}\left(\int_{a}^{b} w(t) d t\right)\left(f(x)+f(a+b-x)+f\left(\frac{a+x}{2}\right)+f\left(\frac{a+2 b-x}{2}\right)\right)-\int_{a}^{b} w(t) f(t) d t .
\end{aligned}
$$

Therefore,

$$
\begin{aligned}
& \left|\frac{1}{4}\left(\int_{a}^{b} w(t) d t\right)\left(f(x)+f(a+b-x)+f\left(\frac{a+x}{2}\right)+f\left(\frac{a+2 b-x}{2}\right)\right)-\int_{a}^{b} w(t) f(t) d t\right| \\
\leq & \frac{1}{2}\left[\int_{a}^{b} w(t) d t\right]\left(D_{2}-D_{1}\right)(b-a) .
\end{aligned}
$$


Corollary 2.1. In Theorem 2.1, if $w$ is a weight function over $[a, b]$ and is symmetric about $\frac{a+b}{2}$, then Inequality (2.3) becomes

$$
\begin{aligned}
& \left|\frac{1}{4}\left(\int_{a}^{b} w(t) d t\right)\left(f(x)+f(a+b-x)+f\left(\frac{a+x}{2}\right)+f\left(\frac{a+2 b-x}{2}\right)\right)-\int_{a}^{b} w(t) f(t) d t\right| \\
\leq & \frac{1}{4}\left[\int_{a}^{b} w(t) d t\right]\left(D_{2}-D_{1}\right)(b-a) .
\end{aligned}
$$

Proof. Since $w$ is symmetric about $\frac{a+b}{2}$, we have

$$
\int_{a}^{b} P_{w}(x, t) d t=0
$$

and

$$
\frac{1}{2} \int_{b}^{a} w(t) d t \leq P_{w}(x, t) \leq \frac{1}{2} \int_{a}^{b} w(t) d t
$$

We can generalize Theorem 2.1 as follows:

Theorem 2.2. Let $-\infty<a<b<\infty$ and $f:[a, b] \rightarrow \mathbb{R}$ be a differentiable function on $(a, b)$. Suppose that $w$ is a weight function over $[a, b]$. If $f^{\prime} \in L^{1}[a, b]$ and $D_{1} \leq f^{\prime}(t) \leq D_{2}$, for all $t \in[a, b]$, where $D_{1}, D_{2}$ are constants, then the inequality

$$
\begin{aligned}
& \left.|| \frac{1}{4}\left(\int_{a}^{b} w(t) d t\right)\left(f(x)+f(a+b-x)+f\left(\frac{a+x}{2}\right)+f\left(\frac{a+2 b-x}{2}\right)\right)-M_{a}^{b}(x) N_{a}^{b}\right] \\
& \quad-\int_{a}^{b} w(t) f(t) d t \mid \\
& \leq \frac{1}{2}\left[\int_{a}^{b} w(t) d t\right]\left(D_{2}-D_{1}\right)(b-a)
\end{aligned}
$$

holds for all $x \in\left[a, \frac{a+b}{2}\right]$, where $M_{a}^{b}(x)=\int_{a}^{b} P_{w}(x, t) d t$ and $N_{a}^{b}=\frac{f(b)-f(a)}{b-a}$.

Proof. By Lemma 2.1, we have

$$
\begin{aligned}
& \frac{1}{b-a} \int_{a}^{b} P_{w}(x, t) f^{\prime}(t) d t-\frac{1}{(b-a)^{2}} \int_{a}^{b} P_{w}(x, t) d t \int_{a}^{b} f^{\prime}(t) d t \\
= & \frac{1}{4(b-a)}\left[\int_{a}^{b} w(t) d t\right]\left[f(x)+f(a+b-x)+f\left(\frac{a+x}{2}\right)+f\left(\frac{a+2 b-x}{2}\right)\right] \\
& -\frac{f(b)-f(a)}{(b-a)^{2}} \int_{a}^{b} P_{w}(x, t) d t-\frac{1}{b-a} \int_{a}^{b} w(t) f(t) d t .
\end{aligned}
$$


By Gruss Inequality, we have

$$
\begin{aligned}
& \left|\frac{1}{b-a} \int_{a}^{b} P_{w}(x, t) f^{\prime}(t) d t-\frac{1}{(b-a)^{2}} \int_{a}^{b} P_{w}(x, t) d t \int_{a}^{b} f^{\prime}(t) d t\right| \\
\leq & \frac{1}{2}\left[\int_{a}^{b} w(t) d t\right]\left(D_{2}-D_{1}\right) .
\end{aligned}
$$

Therefore,

$$
\begin{aligned}
& \left.|| \frac{1}{4}\left(\int_{a}^{b} w(t) d t\right)\left(f(x)+f(a+b-x)+f\left(\frac{a+x}{2}\right)+f\left(\frac{a+2 b-x}{2}\right)\right)-M_{a}^{b}(x) N_{a}^{b}\right] \\
& \quad-\int_{a}^{b} w(t) f(t) d t \mid \\
& \leq \frac{1}{2}\left[\int_{a}^{b} w(t) d t\right]\left(D_{2}-D_{1}\right)(b-a) .
\end{aligned}
$$

Theorem 2.3. Let $f: I \subset \mathbb{R} \rightarrow \mathbb{R}$ be a differentiable mapping on $I^{0}$, the interior of the interval $I$, and let $a, b \in I$ with $a<b$. Let $w$ be a weight function over $[a, b]$ with $\int_{a}^{b} t w(t) d t=\frac{b+a}{2} \int_{a}^{b} w(t) d t$. If $f^{\prime} \in L^{1}[a, b]$ with $D_{1} \leq f^{\prime}(t) \leq D_{2}$ for all $t \in[a, b]$, where $D_{1}, D_{2}$ are constants, then for each $x \in\left[a, \frac{a+b}{2}\right]$, we have

$$
\begin{gathered}
\mid \frac{1}{4}\left[\int_{a}^{b} w(t) d t\right]\left[f(x)+f(a+b-x)+f\left(\frac{a+x}{2}\right)+f\left(\frac{a+2 b-x}{2}\right)\right] \\
-\int_{a}^{b} f(t) w(t) d t\left|\leq \frac{D_{2}-D_{1}}{2} \int_{a}^{b}\right| P_{w}(x, t) \mid d t .
\end{gathered}
$$

Proof. Let $\quad D=\frac{D_{1}+D_{2}}{2}$

Using Identity (2.2) with $f(t)=t$, and the fact that $\int_{a}^{b} t w(t) d t=\frac{b+a}{2} \int_{a}^{b} w(t) d t$, we get that

$$
\int_{a}^{b} P_{w}(x, t) d t=0
$$

which implies that

$$
\int_{a}^{b} P_{w}(x, t) f^{\prime}(t) d t=\int_{a}^{b} P_{w}(x, t)\left(f^{\prime}(t)-D\right) d t .
$$


Using Lemma 2.1, we have

$$
\begin{aligned}
& \int_{a}^{b} P_{w}(x, t)\left(f^{\prime}(t)-D\right) d t \\
= & \frac{1}{4}\left[\int_{a}^{b} w(t) d t\right]\left[f(x)+f(a+b-x)+f\left(\frac{a+x}{2}\right)+f\left(\frac{a+2 b-x}{2}\right)\right]-\int_{a}^{b} f(t) w(t) d t .
\end{aligned}
$$

Note that, for each $t \in[a, b]$,

$$
-\left(\frac{D_{2}-D_{1}}{2}\right)=D_{1}-D \leq f^{\prime}(t)-D \leq D_{2}-D=\frac{D_{2}-D_{1}}{2}
$$

which implies that

$$
\max _{t \in[a, b]}\left|f^{\prime}(t)-D\right| \leq \frac{D_{2}-D_{1}}{2}
$$

Thus,

$$
\begin{aligned}
\left|\int_{a}^{b} P_{w}(x, t)\left(f^{\prime}(t)-D\right) d t\right| & \leq \max _{t \in[a, b]}\left|f^{\prime}(t)-D\right| \int_{a}^{b}\left|P_{w}(x, t)\right| d t \\
& \leq \frac{D_{2}-D_{1}}{2} \int_{a}^{b}\left|P_{w}(x, t)\right| d t
\end{aligned}
$$

which implies that

$$
\begin{gathered}
\mid \frac{1}{4}\left[\int_{a}^{b} w(t) d t\right]\left[f(x)+f(a+b-x)+f\left(\frac{a+x}{2}\right)+f\left(\frac{a+2 b-x}{2}\right)\right] \\
-\int_{a}^{b} f(t) w(t) d t\left|\leq \frac{D_{2}-D_{1}}{2} \int_{a}^{b}\right| P_{w}(x, t) \mid d t .
\end{gathered}
$$

We can generalize Theorem 2.3 as follows:

Theorem 2.4. Let $f: I \subset \mathbb{R} \rightarrow \mathbb{R}$ be a differentiable mapping on $I^{0}$, the interior of the interval $I$, and let $a, b \in I$ with $a<b$. Let $w$ be a weight function over $[a, b]$. If $f^{\prime} \in L^{1}[a, b]$ with $D_{1} \leq f^{\prime}(t) \leq D_{2}$ for all $t \in[a, b]$, where $D_{1}, D_{2}$ are constants, then for each $x \in\left[a, \frac{a+b}{2}\right]$, we have

$$
\begin{gathered}
\mid\left[\frac{1}{4}\left(\int_{a}^{b} w(t) d t\right)\left(f(x)+f(a+b-x)+f\left(\frac{a+x}{2}\right)+f\left(\frac{a+2 b-x}{2}\right)\right)-M_{a}^{b}(x) D\right] \\
-\int_{a}^{b} w(t) f(t) d t\left|\leq \frac{D_{2}-D_{1}}{2} \int_{a}^{b}\right| P_{w}(x, t) \mid d t .
\end{gathered}
$$

where $M_{a}^{b}=\int_{a}^{b} P_{w}(x, t) d t$ and $D=\frac{D_{1}+D_{2}}{2}$. 
Proof. Let $\quad D=\frac{D_{1}+D_{2}}{2}$

Using Lemma 2.1, we have

$$
\begin{aligned}
& \int_{a}^{b} P_{w}(x, t)\left(f^{\prime}(t)-D\right) d t \\
= & \frac{1}{4}\left[\int_{a}^{b} w(t) d t\right]\left[f(x)+f(a+b-x)+f\left(\frac{a+x}{2}\right)+f\left(\frac{a+2 b-x}{2}\right)\right] \\
& -D \int_{a}^{b} P_{w}(x, t) d t-\int_{a}^{b} f(t) w(t) d t .
\end{aligned}
$$

Using same argument as in the proof of Theorem 2.3, we get

$$
\left|\int_{a}^{b} P_{w}(x, t)\left(f^{\prime}(t)-D\right) d t\right| \leq \frac{D_{2}-D_{1}}{2} \int_{a}^{b}\left|P_{w}(x, t)\right| d t
$$

which implies that

$$
\begin{aligned}
& \mid\left[\frac{1}{4}\left(\int_{a}^{b} w(t) d t\right)\left(f(x)+f(a+b-x)+f\left(\frac{a+x}{2}\right)+f\left(\frac{a+2 b-x}{2}\right)\right)-M_{a}^{b}(x) D\right] \\
& -\int_{a}^{b} w(t) f(t) d t\left|\leq \frac{D_{2}-D_{1}}{2} \int_{a}^{b}\right| P_{w}(x, t) \mid d t .
\end{aligned}
$$

Theorem 2.5. $f: I \subset \mathbb{R} \rightarrow \mathbb{R}$ be a differentiable mapping on $(a, b)$. Let $w$ be a weight function over $[a, b]$ with $\int_{a}^{b} t w(t) d t=\frac{b+a}{2} \int_{a}^{b} w(t) d t$. If $f^{\prime} \in L^{1}[a, b]$ with $D_{1} \leq f^{\prime}(t) \leq D_{2}$ for all $t \in[a, b]$, where $D_{1}, D_{2}$ are constants, then for each $x \in\left[a, \frac{a+b}{2}\right]$, we have

$$
\begin{gathered}
\mid \frac{1}{4}\left[\int_{a}^{b} w(t) d t\right]\left[f(x)+f(a+b-x)+f\left(\frac{a+x}{2}\right)+f\left(\frac{a+2 b-x}{2}\right)\right] \\
\quad-\int_{a}^{b} f(t) w(t) d t\left|\leq(b-a)\left(\frac{f(b)-f(a)}{b-a}-D_{1}\right) \sup _{t \in[a, b]}\right| P_{w}(x, t) \mid,
\end{gathered}
$$

and

$$
\begin{gathered}
\mid\left[\frac{1}{4} \int_{a}^{b} w(t) d t\right]\left[f(x)+f(a+b-x)+f\left(\frac{a+x}{2}\right)+f\left(\frac{a+2 b-x}{2}\right)\right] \\
\quad-\int_{a}^{b} f(t) w(t) d t\left|\leq(b-a)\left(D_{2}-\frac{f(b)-f(a)}{b-a}\right) \sup _{t \in[a, b]}\right| P_{w}(x, t) \mid .
\end{gathered}
$$


Proof. Using Identity (2.2) with $f(t)=t$, and the fact that $\int_{a}^{b} t w(t) d t=\frac{b+a}{2} \int_{a}^{b} w(t) d t$, we get that

$$
\int_{a}^{b} P_{w}(x, t) d t=0
$$

which implies that

$$
\begin{aligned}
& \int_{a}^{b} P_{w}(x, t) f^{\prime}(t) d t=\int_{a}^{b} P_{w}(x, t)\left(f^{\prime}(t)-D\right) d t, \\
& \int_{a}^{b} f^{\prime}(t) P_{w}(x, t) d t=\int_{a}^{b} P_{w}(x, t)\left(f^{\prime}(t)-D_{1}\right) d t,
\end{aligned}
$$

and

$$
\int_{a}^{b} f^{\prime}(t) P_{w}(x, t) d t=\int_{a}^{b} P_{w}(x, t)\left(f^{\prime}(t)-D_{2}\right) d t .
$$

Using Lemma 2.1 and the triangle inequality we get

$$
\begin{gathered}
\mid \frac{1}{4}\left[\int_{a}^{b} w(t) d t\right]\left[f(x)+f(a+b-x)+f\left(\frac{a+x}{2}\right)+f\left(\frac{a+2 b-x}{2}\right)\right] \\
-\int_{a}^{b} f(t) w(t) d t\left|\leq \int_{a}^{b}\right| P_{w}(x, t)\left(f^{\prime}(t)-D_{1}\right) \mid d t
\end{gathered}
$$

and

$$
\begin{gathered}
\mid \frac{1}{4}\left[\int_{a}^{b} w(t) d t\right]\left[f(x)+f(a+b-x)+f\left(\frac{a+x}{2}\right)+f\left(\frac{a+2 b-x}{2}\right)\right] \\
-\int_{a}^{b} f(t) w(t) d t\left|\leq \int_{a}^{b}\right| P_{w}(x, t)\left(f^{\prime}(t)-D_{2}\right) \mid d t .
\end{gathered}
$$

Note that

$$
\begin{aligned}
& \int_{a}^{b}\left|P_{w}(x, t) d t\left(f^{\prime}(t)-D_{1}\right)\right| d t \\
\leq & \sup _{t \in[a, b]}\left|P_{w}(x, t)\right| \int_{a}^{b}\left|f^{\prime}(t)-D_{1}\right| d t
\end{aligned}
$$

and

$$
\begin{aligned}
\int_{a}^{b}\left|f^{\prime}(t)-D_{1}\right| d t & =\int_{a}^{b}\left(f^{\prime}(t)-D_{1}\right) d t \\
& =f(b)-f(b)-D_{1}(b-a) \\
& =(b-a)\left[\frac{f(b)-f(b)}{b-a}-D_{1}\right] .
\end{aligned}
$$


Similarly,

$$
\begin{aligned}
& \int_{a}^{b}\left|P_{w}(x, t)\left(f^{\prime}(t)-D_{2}\right)\right| d t \\
\leq & \sup _{t \in[a, b]}\left|P_{w}(x, t)\right| \int_{a}^{b}\left|f^{\prime}(t)-D_{2}\right| d t .
\end{aligned}
$$

and

$$
\begin{aligned}
\int_{a}^{b}\left|f^{\prime}(t)-D_{2}\right| d t & =\int_{a}^{b} D_{2}-f^{\prime}(t) d t \\
& =D_{2}(b-a)-(f(b)-f(b)) \\
& =(b-a)\left[D_{2}-\frac{f(b)-f(b)}{b-a}\right] .
\end{aligned}
$$

Therefore,

$$
\begin{gathered}
\mid \frac{1}{4}\left[\int_{a}^{b} w(t) d t\right]\left[f(x)+f(a+b-x)+f\left(\frac{a+x}{2}\right)+f\left(\frac{a+2 b-x}{2}\right)\right] \\
-\int_{a}^{b} f(t) w(t) d t\left|\leq(b-a)\left(\frac{f(b)-f(a)}{b-a}-D_{1}\right) \sup _{t \in[a, b]}\right| P_{w}(x, t) \mid,
\end{gathered}
$$

and

$$
\begin{gathered}
\mid \frac{1}{4}\left[\int_{a}^{b} w(t) d t\right]\left[f(x)+f(a+b-x)+f\left(\frac{a+x}{2}\right)+f\left(\frac{a+2 b-x}{2}\right)\right] \\
-\int_{a}^{b} f(t) w(t) d t\left|\leq(b-a)\left(D_{2}-\frac{f(b)-f(a)}{b-a}\right) \sup _{t \in[a, b]}\right| P_{w}(x, t) \mid .
\end{gathered}
$$

\subsection{The $L^{2}$ Case.}

Theorem 2.6. Let $f:[a, b] \rightarrow \mathbb{R}$ be an absolutely continuous mapping on $(a, b)$ with $f^{\prime} \in L^{2}[a, b]$. Suppose that $w$ is a weight function over $[a, b]$ with $\int_{a}^{b} t w(t) d t=\frac{b+a}{2} \int_{a}^{b} w(t) d t$. Then for each $x \in\left[a, \frac{a+b}{2}\right]$, we have

$$
\begin{gathered}
\mid \frac{1}{4}\left[\int_{a}^{b} w(t) d t\right]\left[f(x)+f(a+b-x)+f\left(\frac{a+x}{2}\right)+f\left(\frac{a+2 b-x}{2}\right)\right] \\
-\int_{a}^{b} f(t) w(t) d t \mid \leq\left\|P_{w}(x, .)\right\|_{2} \sqrt{\left\|f^{\prime}\right\|_{2}^{2}-\left(\frac{f(b)-f(b)}{b-a}\right)^{2}(b-a) .}
\end{gathered}
$$


Proof. Let

$$
D=\int_{a}^{b} f^{\prime}(y) d y
$$

Using Identity (2.2) with $f(t)=t$, and the fact that $\int_{a}^{b} t w(t) d t=\frac{b+a}{2} \int_{a}^{b} w(t) d t$, we get that

$$
\int_{a}^{b} P_{w}(x, t) d t=0
$$

which implies that

$$
\int_{a}^{b} P_{w}(x, t) f^{\prime}(t) d t=\int_{a}^{b} P_{w}(x, t) d t\left(f^{\prime}(t)-D\right) d t .
$$

Using Lemma 2.1, we have

$$
\begin{gathered}
\mid \frac{1}{4}\left[\int_{a}^{b} w(t) d t\right]\left[f(x)+f(a+b-x)+f\left(\frac{a+x}{2}\right)+f\left(\frac{a+2 b-x}{2}\right)\right] \\
-\int_{a}^{b} f(t) w(t) d t|=| \int_{a}^{b}\left(f^{\prime}(t)-D\right) P_{w}(x, t) d t \mid .
\end{gathered}
$$

By Cauchy Schwartz inequality,

$$
\begin{aligned}
& \left|\int_{a}^{b}\left(f^{\prime}(t)-D\right) P_{w}(x, t) d t\right| \\
\leq & \left(\int_{a}^{b}\left(f^{\prime}(t)-D\right)^{2} d t\right)^{\frac{1}{2}}\left(\int_{a}^{b}\left(P_{w}(x, t)\right)^{2} d t\right)^{\frac{1}{2}} \\
= & \left\|P_{w}(x, .)\right\|_{2}\left(\int_{a}^{b}\left(f^{\prime}(t)-D\right)^{2} d t\right)^{\frac{1}{2}} .
\end{aligned}
$$

Since $f^{\prime} \in L^{2}[a, b]$

$$
\int_{a}^{b}\left(f^{\prime}(t)-D\right)^{2} d t \leq\left\|f^{\prime}\right\|_{2}^{2}-\left(\frac{f(b)-f(b)}{b-a}\right)^{2}(b-a) .
$$

Therefore,

$$
\begin{gathered}
\mid \frac{1}{4}\left[\int_{a}^{b} w(t) d t\right]\left[f(x)+f(a+b-x)+f\left(\frac{a+x}{2}\right)+f\left(\frac{a+2 b-x}{2}\right)\right] \\
-\int_{a}^{b} f(t) w(t) d t \mid \leq\left\|P_{w}(x, .)\right\|_{2} \sqrt{\left\|f^{\prime}\right\|_{2}^{2}-\left(\frac{f(b)-f(b)}{b-a}\right)^{2}(b-a)} .
\end{gathered}
$$


Theorem 2.7. Let $f:[a, b] \rightarrow \mathbb{R}$ be an absolutely continuous mapping on $(a, b)$ with $f^{\prime \prime} \in L_{2}[a, b]$. Suppose that $w$ is a weight function over $[a, b]$ with $\int_{a}^{b} t w(t) d t=\frac{b+a}{2} \int_{a}^{b} w(t) d t$. Then for each $x \in\left[a, \frac{a+b}{2}\right]$, we have

$$
\begin{gathered}
\left|\frac{1}{4}\left[\int_{a}^{b} w(t) d t\right]\left[f(x)+f(a+b-x)+f\left(\frac{a+x}{2}\right)+f\left(\frac{a+2 b-x}{2}\right)\right]-\int_{a}^{b} f(t) w(t) d t\right| \\
\leq \frac{(b-a)}{\pi}\left\|f^{\prime \prime}\right\|_{2}\left\|P_{w}(x, .)\right\|_{2} .
\end{gathered}
$$

Proof. Let

$$
D=f^{\prime}\left(\frac{a+b}{2}\right)
$$

Using Identity (2.2) with $f(t)=t$, and the fact that $\int_{a}^{b} t w(t) d t=\frac{b+a}{2} \int_{a}^{b} w(t) d t$, we get that

$$
\int_{a}^{b} P_{w}(x, t) d t=0
$$

which implies that

$$
\int_{a}^{b} P_{w}(x, t) f^{\prime}(t) d t=\int_{a}^{b} P_{w}(x, t)\left(f^{\prime}(t)-D\right) d t .
$$

Using Lemma 2.1, we have

$$
\begin{gathered}
\mid \frac{1}{4}\left[\int_{a}^{b} w(t) d t\right]\left[f(x)+f(a+b-x)+f\left(\frac{a+x}{2}\right)+f\left(\frac{a+2 b-x}{2}\right)\right] \\
-\int_{a}^{b} f(t) w(t) d t|=| \int_{a}^{b}\left(f^{\prime}(t)-D\right) P_{w}(x, t) d t \mid \\
\leq\left(\int_{a}^{b}\left(f^{\prime}(t)-D\right)^{2} d t\right)^{\frac{1}{2}}\left(\int_{a}^{b}\left(P_{w}(x, t)\right)^{2} d t\right)^{\frac{1}{2}} \\
=\left\|P_{w}(x, .)\right\|_{2}\left(\int_{a}^{b}\left(f^{\prime}(t)-D\right)^{2} d t\right)^{\frac{1}{2}} .
\end{gathered}
$$

By Diaz -Metcalf inequality [16]

$$
\int_{a}^{b}\left(f^{\prime}(t)-D\right)^{2} d t \leq \frac{(b-a)^{2}}{\pi^{2}}\left\|f^{\prime \prime}\right\|_{2}^{2} .
$$

Therefore,

$$
\begin{gathered}
\left|\frac{1}{4}\left[\int_{a}^{b} w(t) d t\right]\left[f(x)+f(a+b-x)+f\left(\frac{a+x}{2}\right)+f\left(\frac{a+2 b-x}{2}\right)\right]-\int_{a}^{b} f(t) w(t) d t\right| \\
\leq \frac{(b-a)}{\pi}\left\|f^{\prime \prime}\right\|_{2}\left\|P_{w}(x, .)\right\|_{2} .
\end{gathered}
$$




\section{Some Applications}

The following Lemma will be useful in calculations.

Lemma 3.1. Let $-\infty<a<b<\infty$ and $w$ be a weight function over $[a, b]$. If $w$ is symmetric about $\frac{a+b}{2}$, then

$(2)$

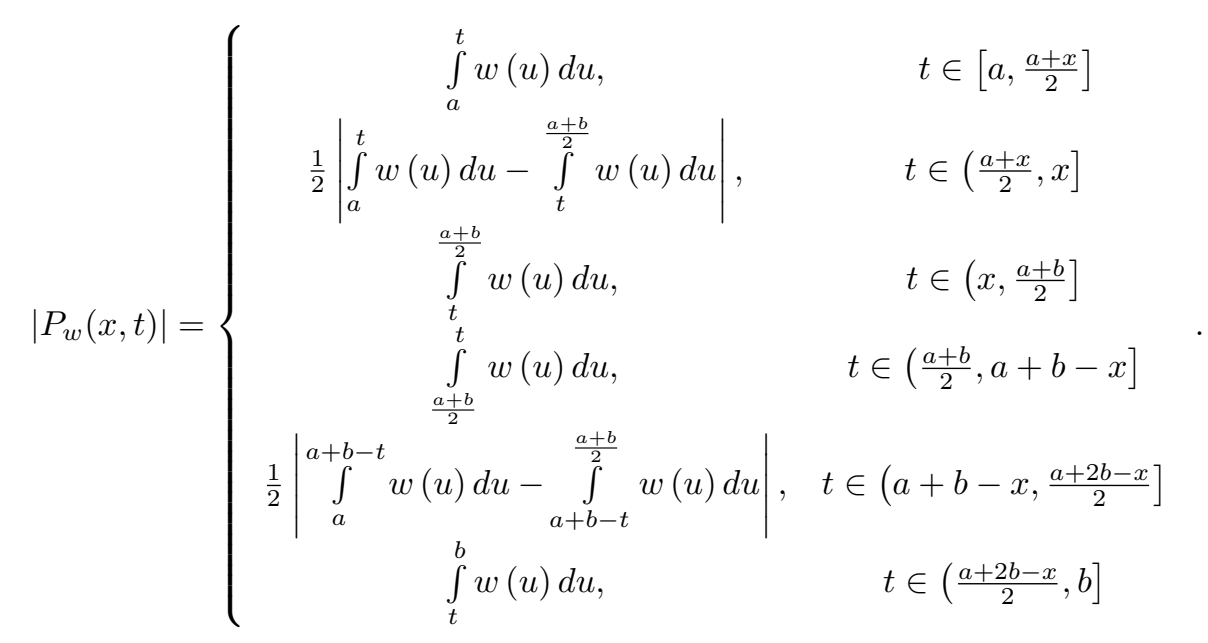

$$
\int_{a}^{b}\left|P_{w}(x, t)\right| d t=2 \int_{a}^{\frac{a+x}{2}} \Phi(t) d t+2 \int_{x}^{\frac{a+b}{2}} \Psi(t) d t+\int_{\frac{a+x}{2}}^{x}|\Phi(t)-\Psi(t)| d t
$$

and

$$
\int_{a}^{b}\left|P_{w}(x, t)\right|^{2} d t=2 \int_{a}^{\frac{a+x}{2}}[\Phi(t)]^{2} d t+2 \int_{x}^{\frac{a+b}{2}}[\Psi(t)]^{2} d t+\frac{1}{2} \int_{\frac{a+x}{2}}^{x}[\Phi(t)-\Psi(t)]^{2} d t
$$

where

$$
\Phi(t)=\int_{a}^{t} w(u) d u \text { and } \Psi(t)=\int_{t}^{\frac{a+b}{2}} w(u) d u, t \in[a, b] .
$$

Remark 3.1. When $w(t)=1$ on $[a, b]$, where $-\infty<a<b<\infty$, we get that

(1)

$$
\int_{a}^{b}\left|P_{w}(x, t)\right| d t=\frac{1}{4}(a+b-2 x)(b-x)
$$

for $x \in\left[a, \frac{3 a+b}{4}\right]$.

(2)

$$
\int_{a}^{b}\left|P_{w}(x, t)\right| d t=\frac{1}{4}(x-a)^{2}+\frac{5}{4}\left(\frac{a+b}{2}-x\right)^{2}+\left(\frac{3 a+b}{4}-x\right)^{2},
$$

for $x \in\left(\frac{3 a+b}{4}, b\right]$. 
Now we give some applications on the results found. Recall that a tagged partition $P$ of a finite interval $[a, b]$ is a finite sequence of numbers $a=x_{0}<x_{1}<\cdots<x_{n}=b$, with corresponding values $t_{i} \in\left[x_{i-1}, x_{i}\right]$, for $i=1, \ldots, n$.

Theorem 3.1. Let $-\infty<a<b<\infty$ and $f:[a, b] \rightarrow \mathbb{R}$ be a differentiable function on $(a, b)$, and $P: a=x_{0}<x_{1}<\cdots<x_{n}=b$ be a tagged partition with corresponding values $t_{i} \in\left[x_{i-1}, \frac{x_{i-1}+x_{i}}{2}\right]$, for $i=1, \ldots, n$. Suppose that $w$ is a weight function over $[a, b]$. If $f^{\prime} \in L^{1}[a, b]$ and $D_{1} \leq f^{\prime}(t) \leq D_{2}$, for all $t \in[a, b]$, where $D_{1}, D_{2}$ are constants, then we have the quadrature formula

$$
\int_{a}^{b} w(t) f(t) d t=A_{w}(f, P)+R_{w}(f, P),
$$

where

$$
\begin{gathered}
A_{w}(f, P)=\sum_{i=0}^{n-1} \frac{1}{4} W_{x_{i}}^{x_{i+1}}\left(f\left(t_{i+1}\right)+f\left(x_{i}+x_{i+1}-t_{i+1}\right)+f\left(\frac{x_{i}+t_{i+1}}{2}\right)\right. \\
\left.+f\left(\frac{x_{i}+2 x_{i+1}-t_{i+1}}{2}\right)\right)-M_{x_{i}}^{x_{i+1}}\left(t_{i+1}\right) N_{x_{i}}^{x_{i+1}}, \\
W_{x_{i}}^{x_{i+1}}=\int_{x_{i}}^{x_{i+1}} w(t) d t, 0 \leq i \leq n-1, \\
M_{x_{i}}^{x_{i+1}}\left(t_{i+1}\right)=\int_{x_{i}}^{x_{i+1}} P_{w}(x, t) d t, 0 \leq i \leq n-1, \\
N_{x_{i}}^{x_{i+1}}=\frac{f\left(x_{i+1}\right)-f\left(x_{i}\right)}{x_{i+1}-x_{i}}, 0 \leq i \leq n-1,
\end{gathered}
$$

and the remainder satisfies the inequality

$$
\left|R_{w}(f, P)\right| \leq \frac{\left(D_{2}-D_{1}\right)}{2} \sum_{i=0}^{n-1} W_{x_{i}}^{x_{i+1}}\left(x_{i+1}-x_{i}\right) .
$$

Proof. For each $0 \leq i \leq n-1$, applying Theorem 2.2 on $\left[x_{i-1}, x_{i}\right]$ with $x=t_{i+1}$, we get that

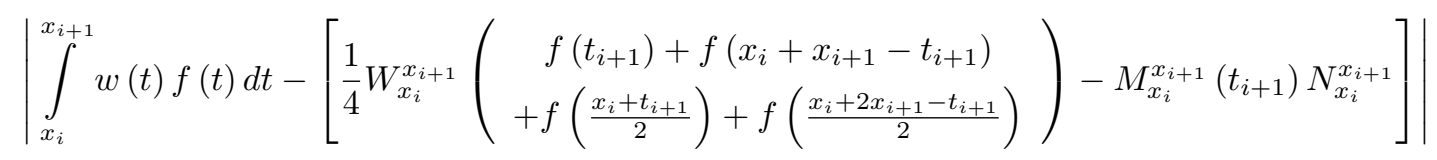

$$
\begin{aligned}
& \leq \frac{\left(D_{2}-D_{1}\right)}{2} W_{x_{i}}^{x_{i+1}}\left(x_{i+1}-x_{i}\right) \text {. }
\end{aligned}
$$

Using the Triangle Inequality, we find that

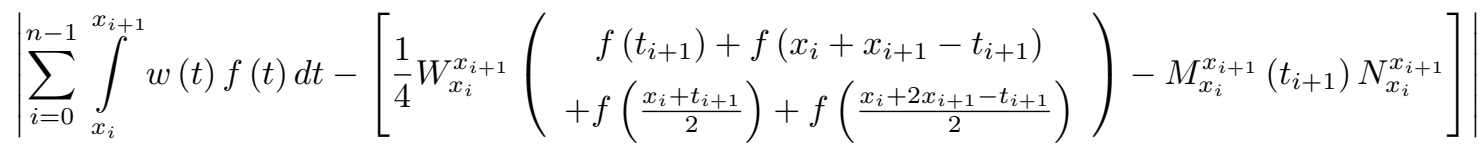

$$
\begin{aligned}
& \leq \frac{\left(D_{2}-D_{1}\right)}{2} \sum_{i=0}^{n-1} W_{x_{i}}^{x_{i+1}}\left(x_{i+1}-x_{i}\right) \text {. }
\end{aligned}
$$


But

$$
\sum_{i=0}^{n-1} \int_{x_{i}}^{x_{i+1}} w(t) f(t) d t=\int_{a}^{b} w(t) f(t) d t
$$

which implies that

$$
\int_{a}^{b} w(t) f(t) d t=A_{w}(f, P)+R_{w}(f, P)
$$

and

$$
\left|R_{w}(f, P)\right| \leq \frac{\left(D_{2}-D_{1}\right)}{2} \sum_{i=0}^{n-1} W_{x_{i}}^{x_{i+1}}\left(x_{i+1}-x_{i}\right) .
$$

Theorem 3.2. Let $f: I \subset \mathbb{R} \rightarrow \mathbb{R}$ be a differentiable mapping on $I^{0}$, the interior of the interval $I$, $a, b \in I$ with $a<b$, and $P: a=x_{0}<x_{1}<\cdots<x_{n}=b$ be a tagged partition with corresponding values $t_{i} \in\left[x_{i-1}, \frac{x_{i-1}+x_{i}}{2}\right]$, for $i=1, \ldots, n$. Suppose that $w$ is a weight function over $[a, b]$. If $f^{\prime} \in L^{1}[a, b]$ with $D_{1} \leq f^{\prime}(t) \leq D_{2}$ for all $t \in[a, b]$, where $D_{1}, D_{2}$ are constants, then we have the quadrature formula

$$
\int_{a}^{b} w(t) f(t) d t=A_{w}(f, P)+R_{w}(f, P),
$$

where

$$
\begin{gathered}
A_{w}(f, P)=\sum_{i=0}^{n-1} \frac{1}{4} W_{x_{i}}^{x_{i+1}}\left(f\left(t_{i+1}\right)+f\left(x_{i}+x_{i+1}-t_{i+1}\right)+f\left(\frac{x_{i}+t_{i+1}}{2}\right)\right. \\
\left.+f\left(\frac{x_{i}+2 x_{i+1}-t_{i+1}}{2}\right)\right)-D M_{x_{i}}^{x_{i+1}}\left(t_{i+1}\right), \\
W_{x_{i}}^{x_{i+1}}=\int_{x_{i}}^{x_{i+1}} w(t) d t, 0 \leq i \leq n-1, \\
M_{x_{i}}^{x_{i+1}}\left(t_{i+1}\right)=\int_{x_{i}}^{x_{i+1}} P_{w}(x, t) d t, 0 \leq i \leq n-1, \\
D=\frac{D_{1}+D_{2}}{2},
\end{gathered}
$$

and the remainder satisfies the inequality

$$
\left|R_{w}(f, P)\right| \leq \frac{\left(D_{2}-D_{1}\right)}{2} \int_{a}^{b}\left|P_{w}(x, t)\right| d t .
$$


Proof. For each $0 \leq i \leq n-1$, applying Theorem 2.4 on $\left[x_{i-1}, x_{i}\right]$ with $x=t_{i+1}$, we get that

$$
\begin{aligned}
& \left|\int_{x_{i}}^{x_{i+1}} w(t) f(t) d t-\left[\frac{1}{4} W_{x_{i}}^{x_{i+1}}\left(\begin{array}{c}
f\left(t_{i+1}\right)+f\left(x_{i}+x_{i+1}-t_{i+1}\right) \\
+f\left(\frac{x_{i}+t_{i+1}}{2}\right)+f\left(\frac{x_{i}+2 x_{i+1}-t_{i+1}}{2}\right)
\end{array}\right)-D M_{x_{i}}^{x_{i+1}}\left(t_{i+1}\right)\right]\right| \\
& \leq \frac{\left(D_{2}-D_{1}\right)}{2} \int_{x_{i}}^{x_{i+1}}\left|P_{w}(x, t)\right| d t .
\end{aligned}
$$

Using the triangle inequality, we find that

$$
\begin{aligned}
& \left|\sum_{i=0}^{n-1} \int_{x_{i}}^{x_{i+1}} w(t) f(t) d t-\left[\frac{1}{4} W_{x_{i}}^{x_{i+1}}\left(\begin{array}{c}
f\left(t_{i+1}\right)+f\left(x_{i}+x_{i+1}-t_{i+1}\right) \\
+f\left(\frac{x_{i}+t_{i+1}}{2}\right)+f\left(\frac{x_{i}+2 x_{i+1}-t_{i+1}}{2}\right)
\end{array}\right)-D M_{x_{i}}^{x_{i+1}}\left(t_{i+1}\right)\right]\right| \\
& \leq \frac{\left(D_{2}-D_{1}\right)}{2} \sum_{i=0}^{n-1} \int_{x_{i}}^{x_{i+1}}\left|P_{w}(x, t)\right| d t
\end{aligned}
$$

But

$$
\sum_{i=0}^{n-1} \int_{x_{i}}^{x_{i+1}} w(t) f(t) d t=\int_{a}^{b} w(t) f(t) d t
$$

and

$$
\sum_{i=0}^{n-1} \int_{x_{i}}^{x_{i+1}}\left|P_{w}(x, t)\right| d t=\int_{a}^{b}\left|P_{w}(x, t)\right| d t
$$

which implies that

$$
\int_{a}^{b} w(t) f(t) d t=A_{w}(f, P)+R_{w}(f, P)
$$

and

$$
\left|R_{w}(f, P)\right| \leq \frac{\left(D_{2}-D_{1}\right)}{2} \int_{a}^{b}\left|P_{w}(x, t)\right| d t .
$$

Before we introduce the next application, recall that if $X$ is a random variable with values in a finite interval $[a, b], a<b$, and $f:[a, b] \rightarrow[0,1]$ is a probability density function, then the cumulative distribution function with respect to $f$ is denoted by $F$ and is defined as:

$$
F(x)=\int_{a}^{x} f(t) d t \text { for each } x \in[a, b] .
$$

Since $f$ satisfies the condition

$$
\int_{a}^{b} f(x) d x=1
$$

we find that $F(b)=1$, and clearly $F(a)=0$. The expectation of $X$ is defined as:

$$
E(X)=\int_{a}^{b} t \frac{d F}{d t} d t
$$


Using integration by parts, we get that

$$
\begin{aligned}
E(X) & =b F(b)-a F(a)-\int_{a}^{b} F(t) d t \\
& =b-\int_{a}^{b} F(t) d t .
\end{aligned}
$$

Theorem 3.3. Let $X$ be a random variable with values in a finite interval $[a, b], a<b$, and $f:[a, b] \rightarrow[0,1]$ be a probability density function. Let $w$ be a differentiable weight function over $[a, b]$ such that $w(b)=1$ and $\int_{a}^{b} t w(t) d t=\frac{b+a}{2} \int_{a}^{b} w(t) d t$. Let $F$ be the cumulative distribution function with respect to $f$. If $f \in L^{1}[a, b]$ and $D_{1} \leq f(t) \leq D_{2}$, for all $t \in[a, b]$, where $D_{1}, D_{2}$ are constants, then the inequality

$$
\begin{aligned}
& \left|\frac{1}{4}\left(\int_{a}^{b} w(t) d t\right)\left(\begin{array}{c}
F(x)+F(a+b-x) \\
+F\left(\frac{a+x}{2}\right)+F\left(\frac{a+2 b-x}{2}\right)
\end{array}\right)-\left(b-E_{G}\right)\right| \\
\leq & \frac{1}{2}\left[\int_{a}^{b} w(t) d t\right]\left(D_{2}-D_{1}\right)(b-a) .
\end{aligned}
$$

holds for all $x \in\left[a, \frac{a+b}{2}\right]$, where

$$
E_{G}=b-\int_{a}^{b} w(t) F(t) d t .
$$

Proof. Define the function $G$ over $[a, b]$ as follows:

$$
G(x)=\int_{a}^{x} \frac{d}{d t}(w F) d t, x \in[a, b] .
$$

Note that

$$
G(a)=0
$$

and

$$
\begin{aligned}
G(b) & =\int_{a}^{b} \frac{d}{d t}(w F) d t \\
& =w(b) F(b)-w(a) F(a) \\
& =1 .
\end{aligned}
$$

Let

$$
E_{G}=\int_{a}^{b} t \frac{d}{d t}(w F) d t
$$

Using integration by parts, we get that

$$
\begin{aligned}
E_{G} & =b w(b) F(b)-a w(a) F(a)-\int_{a}^{b} w(t) F(t) d t \\
& =b-\int_{a}^{b} w(t) F(t) d t
\end{aligned}
$$

which implies that

$$
\int_{a}^{b} w(t) F(t) d t=b-E_{G} .
$$


Applying Theorem 2.1 on $F$, we get that

$$
\begin{gathered}
\mid \frac{1}{4}\left(\int_{a}^{b} w(t) d t\right)\left(F(x)+F(a+b-x)+F\left(\frac{a+x}{2}\right)+F\left(\frac{a+2 b-x}{2}\right)\right) \\
-\int_{a}^{b} w(t) F(t) d t \mid \leq \frac{1}{2}\left[\int_{a}^{b} w(t) d t\right]\left(D_{2}-D_{1}\right)(b-a),
\end{gathered}
$$

which implies that

$$
\begin{aligned}
& \left|\frac{1}{4}\left(\int_{a}^{b} w(t) d t\right)\left(\begin{array}{c}
F(x)+F(a+b-x) \\
+F\left(\frac{a+x}{2}\right)+F\left(\frac{a+2 b-x}{2}\right)
\end{array}\right)-\left(b-E_{G}\right)\right| \\
& \leq \frac{1}{2}\left[\int_{a}^{b} w(t) d t\right]\left(D_{2}-D_{1}\right)(b-a) .
\end{aligned}
$$

\section{REFERENCES}

[1] M.W. Alomari, A companion of ostrowski's inequality for mappings whose first derivatives are bounded and applications numerical integration, Kragujevac J. Math. 36 (2012), 77-82.

[2] N.S. Barnett, S.S. Dragomir and I. Gomma, A companion for the Ostrowski and the generalized trapezoid inequalities, J. Math. Comput. Model. 50 (2009), 179-187.

[3] S.S. Dragomir, Some companions of Ostrowski's inequality for absolutely continuous functions and applications, Bull. Korean Math. Soc. 40(2) (2005), 213-230.

[4] S.S. Dragomir and S. Wang, An inequality of Ostrowski-Grüss type and its applications to the estimation of error bounds for some special means and for some numerical quadrature rules, Comput. Math. Appl. 33(11) (1997), 15-20.

[5] Z. Liu, Some companions of an Ostrowski type inequality and applications, J. Inequal. Pure Appl. Math. 10(2) (2009), $10-12$.

[6] D.S. Mitrinvić, J.E. Pecarić and A.M. Fink, Classical and New Inequalities in Analysis, Kluwer Academic Publishers, Dordrecht, (1993).

[7] D.S. Mitrinović, J.E. Pecarić and A.M. Fink, Inequalities involving functions and their integrals and derivatives, Mathematics and its Applications. (East European Series), Kluwer Acadamic Publications Dordrecht, Vol. 53., (1991).

[8] A. Ostrowski, Über die Absolutabweichung einer differentienbaren Funktionen von ihren Integralimittelwert, Comment. Math. Hel. 10 (1938), 226-227.

[9] A. Qayyum and S. Hussain, A new generalized Ostrowski Grüss type inequality and applications, Appl. Math. Lett. 25 (2012), 1875-1880.

[10] A. Qayyum, M. Shoaib, A.E. Matouk and M.A. Latif, On New Generalized Ostrowski Type Integral inequalities, Abstr. Appl. Anal. 2014 (2014), Art. ID 275806.

[11] A. Qayyum, M. Shoaib and M. A. Latif, A generalized inequality of ostrowski type for twice differentiable bounded mappings and applications, Appl. Math. Sci. 8(38) (2014), 1889-1901.

[12] A. Qayyum, I. Faye, M. Shoaib and M.A. Latif, A Generalization of Ostrowski type inequality for mappings whose second derivatives belong to $L_{1}(a, b)$ and applications, Int. J. Pure Appl. Math. 98(2) (2015), 169-180. 
[13] A. Qayyum, M. Shoaib and I. Faye, Some New Generalized Results on Ostrowski Type Integral Inequalities With Application, J. Comput. Anal. Appl. 19(4) (2015), 693-712.

[14] A. Qayyum, M. Shoaib and I. Faye, On New Weighted Ostrowski Type inequalities Involving Integral Means over End Intervals and Application, Turk. J. Anal. Number Theory, 3(2) (2015), 61-67.

[15] A. Qayyum, M. Shoaib and I. Faye, A Companion of Ostrowski Type Integral Inequality Using a 5-Step Kernel with Some Applications, Filomat, 30(13) (2016), 3601-3614.

[16] N. UJević, New bounds for the first inequality of Ostrowski-Grüss type and applications, Comput. Math. Appl. 46 (2003), 421-427. 